\title{
Osimertinib plus Bevacizumab Improve the Outcome of Leptomeningeal Metastasis Patients with EGFR Mutant Non-Small-Cell Lung Cancer
}

yali yi ( $\sim 353007210011 @ e m a i l . n c u . e d u . c n)$

Nanchang University Second Affiliated Hospital

Jing Cai

Nanchang University Second Affiliated Hospital

Peng Xu

Nanchang University Second Affiliated Hospital

Le Xiong

Nanchang University Second Affiliated Hospital

Zhiqin Lu

Nanchang University Second Affiliated Hospital

\section{Zhimin Zeng}

Nanchang University Second Affiliated Hospital

Anwen Liu

Nanchang University Second Affiliated Hospital https://orcid.org/0000-0001-5279-1068

\section{Research Article}

Keywords: leptomeningeal metastasis, EGFR-mutant, NSCLC, osimertinib, bevacizumab

Posted Date: January 31st, 2022

DOl: https://doi.org/10.21203/rs.3.rs-1243315/v1

License: (c) (i) This work is licensed under a Creative Commons Attribution 4.0 International License. Read Full License 


\section{Abstract \\ Background}

EGFR-mutant non-small cell lung cancer (NSCLC) is prone to leptomeningeal metastasis (LM) after Tyrosine kinase inhibitors (TKIs) treatment. Our previous study suggested that osimertinib plus bevacizumab was safe and effective in LM from EGFR-mutant lung cancer. This study aimed to compare the efficacy of osimertinib plus bevacizumab with osimertinib in EGFR-mutant NSCLC patients with LM.

\section{Methods}

We retrospectively reviewed data from 27 LM patients with EGFR-mutant lung cancer who received osimertinib with or without bevacizumab at the Second Affiliated Hospital of Nanchang University. Next, we investigated the antitumor efficacy of osimertinib plus bevacizumab in an LM xenograft model using the H1975 (EGFR exon20 T790M and exon21 L858R) cell line. We examined the ability of osimertinib plus bevacizumab compared with osimertinib to penetrate the blood-brain barrier (BBB) and explored the potential mechanism.

\section{Results}

Our retrospective study observed the improved survival of LM patients in osimertinib plus bevacizumab group. The median overall survival (OS) of the patients who received osimertinib and bevacizumab ( $\mathrm{n}=$ 16) compared with osimertinib $(n=11)$ was 18.0 months versus 13.7 months (log-rank test, $p=0.046$, $\mathrm{HR}=2.867,95 \% \mathrm{Cl}: 1.007-8.162$ ). The median intracranial Progression-free Survival (iPFS) was 10.6 months versus 5.5 months (log-rank test, $p=0.037, \mathrm{HR}=3.401,95 \% \mathrm{Cl}: 1.079-10.720$ ). In the LM xenograft model with $\mathrm{H} 1975$ cells, the combined treatment significantly increased the effective intracranial concentration of osimertinib, modulated the level of E-cadherin and downregulated the levels of EGFR and downstream signaling pathways including p-AKT and reduced tumor microvessel density (TMD), indicated that combined osimertinib with bevacizumab play a synergistic effect in EGFR-mutant LM model possibly by modulating the level of E-cadherin.

\section{Conclusions}

Our findings indicate that osimertinib plus bevacizumab tend to improve the outcome of LM patients with EGFR-mutant NSCLC than osimertinib.

\section{Introduction}

Non-small cell lung cancer (NSCLC) is one of the leading causes of cancer death worldwide[1]. EGFRmutant lung adenocarcinoma is prone to leptomeningeal metastasis (LM) after first-generation TKI 
treatment $[2,3]$. Patients with LM have a poor prognosis and low quality of life[4, 5]. Approximately $3-5 \%$ of patients with NSCLC will develop LM, and the median OS is approximately $4.5-11$ months[6, 7]. Osimertinib, a third-generation EGFR TKI, is currently the standard therapy for lung cancer metastases in the central nervous system (CNS)(including brain/leptomeningeal metastases)[8], which extended the OS to 18.8 months in LM patients with NSCLC $[4,9]$. The incidence of LM in patients with lung cancer, especially in patients with EGFR mutations, is increasing with the emergence of new targeted drugs[1].

TKIs combined with antiangiogenic drugs such as bevacizumab have shown efficacy in lung cancer[10, 11]. A phase II clinical study showed that osimertinib plus bevacizumab is beneficial in the treatment of lung cancer brain metastases[12], and our previous study indicated that osimertinib plus bevacizumab was safe and effective for the treatment of LM in EGFR-mutant lung cancer [NCT04148898][13]. LM is different from brain parenchymal metastasis, and its mechanism and treatment are complicated issues in current clinical treatment. There are currently no research data on the efficacy of osimertinib plus bevacizumab compared with osimertinib in LM.

This is the first study to compare the efficacy of osimertinib plus bevacizumab with osimertinib in LM with EGFR-mutant NSCLC through pre-clinical experiment. Aims to investigate the efficacy and potential mechanisms of osimertinib plus bevacizumab in LM with EGFR-mutant NSCLC.

\section{Materials And Methods 2.1. Patients}

We retrospectively reviewed the charts of 27 patients diagnosed with LM from EGFR-mutant NSCLC who received osimertinib with or without bevacizumab at the Second Affiliated Hospital of Nanchang. The date of LM diagnosis was defined as the date of first CSF cytology examination revealing malignant cells or the date of first MRI (brain or spine) demonstrating LM. The assessment of LM response was based on the modified RANO LM radiological criteria, and the CNS and extra-CNS response was evaluated according to the Response Evaluation Criteria in Solid Tumors (RECIST) version 1.1: comprehensive evaluation according to the improvement of clinical symptoms, the performance of MRI and the clearance of CSF tumor cells[14]. Given that lumbar puncture is an invasive procedure, the response criteria of LM were judged according to the improvement of clinical symptoms and the performance of MRI in our study. All LM patients underwent 1.5T whole brain and spinal cord enhanced MRI scan at baseline, and the MRI scan thickness was $1 \mathrm{~mm}$, bravo and cube sequence having a high sensitivity and specificity in the diagnosis of LM. The Overall survival (OS) was defined as the time between the initiation of diagnosis to the date of death or last follow-up by November 5, 2020. The intracranial Progression-free Survival (iPFS) was defined as the time from the diagnosis of LM to the disease progression or death. Four weeks after the initiation of Osimertinib and bevacizumab, neurological evaluations, brain MRI and chest/abdominal computed tomography were routinely performed and were then performed every 1 months. The main endpoint of this study was iPFS. 
The eligibility criteria were as follows: (i) age 18-80 years; (ii) histologically or cytologically confirmed NSCLC; (iii) the detection of an EGFR mutation, with EGFR status identified from primary lung tumors using the amplification refractory mutation system (ARMS) or next-generation sequencing (NGS) analysis; (iv) LM defined by CSF positivity for malignant cells and/or focal or diffuse enhancement of leptomeninges, nerve roots or the ependymal surface diagnosed by magnetic resonance imaging (MRI) with gadolinium contrast; (v) Patients receiving osimertinib plus bevacizumab or osimertinib. (vi) Patients without history of treatment with osimertinib before a diagnosis of LM. Patients who did not meet these criteria were excluded. Clinical outcomes were compared with the Kaplan-Meier log-rank test. This study was approved by the Institutional Review Board of the Second Affiliated Hospital of Nanchang University.

\subsection{Cell Culture}

H1975 cells (EGFR exon20 T790M and exon21 L858R) were purchased from Pro-cell in 2020 (Wuhan, China, was identified by STR) and maintained in RPMI-1640 medium supplemented with $10 \%$ Fetal Bovine Serum and $1 \%$ antibiotic solution in a humidified incubator with $5 \% \mathrm{CO}_{2}$. All reference compounds were purchased from Gibco.

H1975 cells were stably transfected with a GV260 (GeneChem, Shanghai, China) vector containing luciferase, and bioluminescence signals were measured by an in vivo imaging system. H1975-luc tumor cells were prepared for injection after trypsinization and washing with PBS. A viable cell count was performed with trypan blue to adjust the cell concentration to $2 \times 10^{6}$ cells in PBS for each injection.

\subsection{Animal model of LM}

BALB/C nude female mice (6-8 weeks) were obtained from institutional animal breeding services (the Nanchang Royo Biotech Co., Ltd.). The animals were group housed five a cage in a temperaturecontrolled room on a 12-h/12-h light/dark cycle with unlimited access to food and water. All animal procedures in this study were approved by the Animal Care and Use Committee of Nanchang University, and ethical approval was obtained from the Institutional Review Board of the Second Affiliated Hospital of Nanchang University.

Mice were anesthetized with $4 \%$ isoflurane. The skull was exposed with a skin incision $(1 \mathrm{~cm})$ under sterile conditions (ethanol skin wipe) to locate the bregma. A Hamilton syringe needle with H1975-luc human NSCLC cells was injected into the right lateral ventricle (anteroposterior $2.0 \mathrm{~mm}$ from the bregma; lateral $0.2 \mathrm{~mm}$ to the right; and dorsoventral $4 \mathrm{~mm}$ ) at $2 \mu \mathrm{l}$ per min. A total volume of $5 \mu \mathrm{l}$ of cell suspension was injected $[15,16]$. The tumor burden of intracranial lesions and the tumor size in the pia mater were measured using a BLI technique with an in vivo imaging system, MRI and H\&E staining.

\subsection{In Vivo Pharmacodynamic Study}

After confirming tumor formation, the mice were randomly divided into 4 groups ( $n=9$ per group): the control( $0.9 \%$ normal saline, daily, oral), osimertinib (25 mg/ $\mathrm{kg}$, daily, oral)[17], and bevacizumab (5 mg/ $\mathrm{kg}$ twice a week, i.p.)[18], and osimertinib plus bevacizumab. Brain tissues were collected at 1, 6, 12, and 24 $\mathrm{h}$ after a single dose of treatment to determine the penetration of osimertinib in the brain with a validated 
liquid chromatography-tandem mass spectrometry method(n=3). Furthermore, tumor tissues were collected in formalin or frozen at the endpoint of experiments, EGFR downstream signals were evaluated by immunoblotting, and an angiogenesis marker (CD31) was evaluated by immunofluorescence. Once the nude mice lost more than $20 \%$ of their weight, the experiment reached the endpoint, and the mice were euthanized by intraperitoneal injection of pentobarbital sodium $(150 \mathrm{mg} / \mathrm{kg})$. Each experimental group has at least 9 mice, and a total of 40 mice are included.

\subsection{Formaldehyde perfusion of the brain}

To keep the mouse brain tissue as intact as possible, this experiment used formaldehyde perfusion to fix the brain. The mice were anesthetized by the intraperitoneal injection of $1 \%$ pentobarbital sodium( 50 $\mathrm{mg} / \mathrm{kg}$ ), the chest skin was cut open with scissors, the heart was exposed, a syringe needle was inserted into the left ventricle from the left apex towards the aorta, the needle was fixed with hemostatic forceps, physiological saline was quickly perfused until the liver tissue color turned gray, and then paraformaldehyde was slowly perfused. The mouse limbs twitched, and the whole body became stiff. After successful perfusion, the mouse brain tissue was removed and fixed with paraformaldehyde for subsequent experiments.

\subsection{Histology}

\subsubsection{H\&E staining and immunofluorescence}

Mouse brain tissues were quickly excised, immersed in $10 \%$ paraformaldehyde and embedded in paraffin. Brain tissues were cut into $5-\mu \mathrm{m}$ thick sections. Slides were stained with hematoxylin and eosin (H\&E). Pictures were obtained with $\times 10$ and $\times 40$ objective respectively, the pathology diagnosis was completed by two independent pathology doctors. Tumor microvessel density (TMD) was measured by staining for CD31 (Proteintech, Wuhan, China), as previously reported[19]. Briefly, TMD was assessed in hot spots of brain tissue cross-sections identified by light microscopy. Five equal areas were then photographed with a $40 x$ objective (400x magnification). The staining was scored by two independent and experienced pathologists and calculated as the product of the staining intensity. The areas and integrated optical density (IOD) of the slides were analyzed by Image-Pro Plus 6.0 software.

\subsubsection{Western blotting}

Western blotting was performed according to standard methods, as described previously[19]. The tumor sections in brain tissue were snap-frozen in liquid nitrogen for protein isolation, and then tumors were homogenized with a mortar and pestle and lysed in RIPA buffer containing Halt protease \& phosphatase inhibitor cocktail. Soluble proteins were quantified by a BCA protein level detection kit and then subjected to SDS-PAGE followed by immunoblotting. Antibody incubation was conducted overnight at $4^{\circ} \mathrm{C}$. Antibodies included EGFR(1:1000, Servicebio, GB11084-2), AKT (1:1000, Servicebio, GB11689), S473 pAKT (1:1000, Affinity, AF0832), ERK (1/2) (1:1000, Servicebio, GB11560), p-ERK (1/2) (1:1000, Cell Signaling Technology, \#4370), E-cadherin (1:1000, Servicebio, GB14076), HIF-1a (1:1000, Servicebio, GB111339), ACTIN (1:3000, Servicebio, GB12001). Secondary antibodies were diluted 1:5000, and 
immunoreactive proteins were visualized using a Western blotting machine (Thermo) and analyzed with ImageJ software.

\subsubsection{IHC}

Tumor-bearing brain tissue were collected from mice of each group. Tissues were fixed in 10\% buffered formalin for 24 hours following standard procedure for processing, paraffin-embedding, and sectioning to assess PCNA (1:200, Servicebio, GB11010), E-cadherin (1:200, Servicebio, GB14076) by IHC assay. The reaction was visualized using the Servicebio image analysis system, the staining was scored by two independent and experienced pathologists and calculated as the product of the staining intensity. first divides the positive grade: negative without staining, score 0 , weak positive light yellow, score 1, medium positive brown, score 2, strong positive brown, score 3 points. Then analyze and calculate the area of weak, medium, and strong positive in the measurement area, the tissue area of the measurement area, the cumulative optical density value of the positive and the positive area. PCNA and E-cadherin quantification for each sample was determined and molecular data using modified H-scores ([\{\% of weak staining $\} \times 1]+[\{\%$ of moderate staining $\} \times 2]+[\{\%$ of strong staining $\} \times 3])$, to determine the overall percentage of PCNA and E-cad positivity across the entire stained sample, yielding a range from 0-300[20, 21].

\subsection{Statistics}

Preclinical data are presented as the mean \pm SD. Data were analyzed by one-way ANOVA, followed by Dunnett's test or Student's t-test. Survival analysis of animal was performed using a Kaplan-Meier survival curve and a log-rank test. The Kaplan-Meier method was used to estimate and graphically present OS and iPFS. All analyses were performed using GraphPad Prism 7.0 (GraphPad Prism Software, Inc) or IBM SPSS statistics 22. Experiments were repeated independently at least three times. $p<0.05$ was considered statistically significant.

\section{Results}

\subsection{Osimertinib plus bevacizumab improved the efficacy of LM patients with EGFR-mutant NSCLC.}

A total of 70 patients diagnosed with lung cancer and LM in at the Second Affiliated Hospital of Nanchang University from October 2017 to March 2020 were collected. 27 patients with EGFR mutationpositive NSCLC with LM were selected according to the inclusion criteria (Table 1). Enrolled patients received osimertinib $80 \mathrm{mg}$ orally daily with or without bevacizumab $7.5 \mathrm{mg} / \mathrm{kg}$ intravenously every three weeks. Treatment continued until the disease progressed, unacceptable adverse events occurred, or the patient withdrew consent. The pathological type of all patients is lung adenocarcinoma. In these patients, EGFR mutations included L858R (51.8\%) (1 T790M), exon 19 deletion (48\%) (2 T790M). The median follow-up time was 19.1 months. In osimertinib group, Of the 11 total patients, 81.8\% (9) had clinical response, 3 of patients achieved partial response (PR) (27.3\%),6 of patients had stable disease (SD), 2 of 
patients had progressive disease (PD). In osimertinib plus bevacizumab group, Of the 16 total patients, $81.3 \%$ (13) had a clinical response, 6 of patients achieved PR (37.5\%),7 of patients had SD, 3 of patients had PD. The assessments of response to osimertinib or osimertinib plus bevacizumab in 27 LM patients were shown in supplementary table 1 and 2 . The Swimmer plot was accordance with treatment duration of $27 \mathrm{LM}$ patients (Fig. 1A). There is an increasing of median OS of the patients who received osimertinib and bevacizumab ( $n=16,18.0$ months) as compared with patients with Osimertinib treatment $(n=11$, 13.7 months) (log-rank test, $p=0.046, \mathrm{HR}=2.867,95 \% \mathrm{Cl}: 1.007-8.162)$. The median iPFS is 10.6 months versus 5.5 months (log-rank test, $p=0.037, \mathrm{HR}=3.401,95 \% \mathrm{Cl}: 1.079-10.720$ ) (Fig. 1B and C). These data suggested that osimertinib plus bevacizumab improve the survival time of patients with LM from EGFR mutant NSCLC. 


\begin{tabular}{|c|c|c|}
\hline Characteristics & $\begin{array}{l}\text { Osimertinib } \\
(n=11) \%\end{array}$ & $\begin{array}{l}\text { Osimertinib } \\
\text { and bevacizumab } \\
(n=16) \%\end{array}$ \\
\hline Age (median) & $47-70(56)$ & $46-75(60)$ \\
\hline Gender & $4(36.4)$ & $8(50.0)$ \\
\hline Female & $7(63.6)$ & $8(50.0)$ \\
\hline \multicolumn{3}{|l|}{ Male } \\
\hline History of tobacco exposure & $6(54.5)$ & $7(43.8)$ \\
\hline Yes & $5(45.5)$ & $9(56.2)$ \\
\hline \multicolumn{3}{|l|}{ No } \\
\hline ECOG performance status score & $9(81.8)$ & $11(68.8)$ \\
\hline$\geq 2$ & $2(18.2)$ & $5(31.2)$ \\
\hline \multicolumn{3}{|l|}{$<2$} \\
\hline EGFR mutation & 6(54.5) (1 T790M) & $8(50.0)$ \\
\hline Exon $21 \mathrm{~L} 858 \mathrm{R}$ & $5(45.5)(1 \mathrm{~T} 790 \mathrm{M})$ & $8(50.0)(1 \mathrm{~T} 790 \mathrm{M})$ \\
\hline \multicolumn{3}{|l|}{ Exon 19 deletion } \\
\hline Neurological symptoms & 10(90.9) & 15(93.8) \\
\hline Yes & $1(9.1)$ & $1(6.2)$ \\
\hline \multicolumn{3}{|l|}{ No } \\
\hline Exclusively diagnosed LM by CSF cytology & $5(45.5)$ & $8(50.0)$ \\
\hline Yes & $6(54.5)$ & $8(50.0)$ \\
\hline \multicolumn{3}{|l|}{ No } \\
\hline Exclusively diagnosed LM by MRI & $9(81.8)$ & $12(75.0)$ \\
\hline Positive & 2(18.2) & $4(25.0)$ \\
\hline Negative & & \\
\hline
\end{tabular}

CSF, cerebrospinal fluid; ECOG, Eastern Cooperative Oncology Group; EGFR, epidermal growth factor receptor; LM, leptomeningeal metastasis; MRI, magnetic resonance imaging; TKIs, tyrosine kinase inhibitors. osi \pm beva, osimertinib with or without bevacizumab; ITC, intrathecal chemotherapy; WBRT, whole-brain radiotherapy. 


\begin{tabular}{|c|c|c|}
\hline Characteristics & $\begin{array}{l}\text { Osimertinib } \\
(n=11) \%\end{array}$ & $\begin{array}{l}\text { Osimertinib } \\
\text { and bevacizumab } \\
(n=16) \%\end{array}$ \\
\hline Both positive for MRI and CSF cytology & $5(45.5)$ & $5(31.2)$ \\
\hline Yes & $6(54.5)$ & 11(68.8) \\
\hline \multicolumn{3}{|l|}{ No } \\
\hline Systemic therapy before LM diagnosis & 10(90.9) & 14(87.5) \\
\hline First generation TKIs & $1(9.1)$ & $2(12.5)$ \\
\hline Yes & $1(9.1)$ & $3(18.7)$ \\
\hline No & 10(90.9) & 13(81.3) \\
\hline Chemotherapy & 2(18.2) & $2(12.5)$ \\
\hline Yes & $9(81.8)$ & 14(87.5) \\
\hline \multicolumn{3}{|l|}{ No } \\
\hline \multicolumn{3}{|l|}{ Brain radiotherapy } \\
\hline \multicolumn{3}{|l|}{ Yes } \\
\hline \multicolumn{3}{|l|}{ No } \\
\hline Subsequent treatments after osi \pm beva & $1(9.1)$ & $3(18.8)$ \\
\hline Osimertinib + ITC & $0(0.0)$ & $1(6.2)$ \\
\hline Osimertinib +WBRT & 2(18.2) & $1(6.2)$ \\
\hline Chemotherapy & $1(9.1)$ & $0(0.0)$ \\
\hline Osimertinib 160mg & $7(63.6)$ & 11(68.8) \\
\hline \multicolumn{3}{|l|}{ Continued prior treatments } \\
\hline \multicolumn{3}{|c|}{$\begin{array}{l}\text { CSF, cerebrospinal fluid; ECOG, Eastern Cooperative Oncology Group; EGFR, epidermal growth factor } \\
\text { receptor; LM, leptomeningeal metastasis; MRI, magnetic resonance imaging; TKIs, tyrosine kinase } \\
\text { inhibitors. Ositbeva, osimertinib with or without bevacizumab; ITC, intrathecal chemotherapy; WBRT } \\
\text { whole-brain radiotherapy. }\end{array}$} \\
\hline
\end{tabular}

To determine the efficacy of osimertinib plus bevacizumab in LM in EGFR-mutant NSCLC, we constructed a model of LM with lateral ventricle injection (Fig. 2A), and the lung cancer LM xenograft model was confirmed by IVIS imaging, MRI and H\&E (Fig. 2B, C and E). Mass spectrometry analysis showed that the average concentration of osimertinib in the brain tissue in combined group was higher than osimertinib 
group [ 803.2 vs $606.5 \mathrm{ng} / \mathrm{ml}$ (1 hour after treatments), 359.6 vs $193.8 \mathrm{ng} / \mathrm{ml}$ ( 6 hour), $91.8 \mathrm{vs} 31.2 \mathrm{ng} / \mathrm{ml}$ (12 hour), 3.8 vs $1.0 \mathrm{ng} / \mathrm{ml}$ (24 hour) ( $\mathrm{n}=3$ per group, $p<0.05$ )] (Fig. 2D). These results suggested that when osimertinib is combined with bevacizumab, the concentration of osimertinib in the mouse brain was increased.

\subsection{Osimertinib plus bevacizumab effectively inhibits the growth of EGFR-mutant LM xenografts in nude mice}

The effects of osimertinib plus bevacizumab in the LM xenograft model were validated. Within 2 weeks of treatment, the three groups had different degrees of tumor regression except for the progression of the control group compared to baseline (Fig. 3A and B). The body weight change and survival curves implied that the tumor regression of the combined group was superior to that of the osimertinib group after 3 weeks of treatment. The mice in the bevacizumab group lost a significant amount of weight and died quickly, similar to mice in the control group. ( $n=6$ per group, $p<0.05$ ) (Fig. 3C and F). Compared with that in the osimertinib group, the tumors in the osimertinib plus bevacizumab group were significantly regressed (Fig. 3D and G). These results suggested that the combination effectively inhibits the growth of EGFR-mutant LM tumors in vivo.

\subsection{Osimertinib plus bevacizumab suppresses the EGFR downstream signaling pathway and modulates E-cadherin levels in EGFR-mutant LM model mice}

The EGFR downstream signaling pathway was examined in tumor tissues by Western blotting. The results showed that $\mathrm{p}$-AKT and EGFR were significantly decreased in the osimertinib plus bevacizumab group compared with the other groups. $(n=3, p<0.05)$ (Fig. 4A and B (a-e)). Meanwhile, our data showed that TMD was significantly decreased in the osimertinib and bevacizumab group compared with that in the other groups $(n=3, p<0.05)$ (Fig. $4 \mathrm{C}$ and $B(f)$ ). These results further verified that osimertinib and bevacizumab could play a synergistic effect in EGFR-mutant LM model. To understand the potentially mechanism of the combination treatment, we assessed PCNA, E-cadherin, ADAM9 and HIF-1a levels in xenograft tumors receiving the treatments. The combination did not affect the levels of ADAM9 and HIF$1 \mathrm{a}$, but the E-cad levels was more significantly decreased in combination group compared with osimertinib group $(n=3, p<0.05)$ (Fig. 5). Hence, we demonstrated the in vivo modulation of E-cadherin by the combination of osimertinib and bevacizumab.

\section{Discussion}

Due to the unclear mechanism of LM and the existence of the blood-brain barrier, it is difficult for drugs to reach an effective intracranial concentration. The treatment of LM in lung cancer is still a complicated problem. This is the first study to compare the effects of osimertinib with or without bevacizumab in LM of EGFR-mutant NSCLC. The efficacy is compared through a combination of basic research and clinical analysis and its possible mechanism is explored. 
Studies have shown that EGFR-TKIs (erlotinib) and angiogenesis inhibitors that target endothelial growth factor receptor (anti-VEGFR) (bevacizumab) (A+T) achieved superior PFS and acceptable safety in NSCLC patients with intracranial metastasis[22]. Our study retrospectively analyzed 27 LM patients with EGFR-mutant lung cancer who received osimertinib with or without bevacizumab, the median OS of osimertinib plus bevacizumab group $(n=16)$ compared osimertinib group $(n=11)$ was 18.0 months versus 13.7 months (log-rank test, $p=0.046, \mathrm{HR}=2.867,95 \% \mathrm{Cl}: 1.007-8.162$ ). The median iPFS is 10.6 months versus 5.5 months (log-rank test, $p=0.037, \mathrm{HR}=3.401,95 \% \mathrm{Cl}: 1.079-10.720$ ). Then we investigated the antitumor effects of osimertinib and bevacizumab in an EGFR-mutant LM model. We found that osimertinib plus bevacizumab significantly improved the concentration of osimertinib in mouse brain tissue.

A series of studies have supported that $A+T$ therapy can result in improved survival benefits[23]. The J025567 recommended combined erlotinib and bevacizumab as a first-line regimen in EGFR mutationpositive NSCLC[24, 25]. The NEJ026 also suggested that TKIs combined with bevacizumab extended PFS 3.6 months compared to erlotinib in EGFR-mutant NSCLC[11]. The PFS of NSCLC patients with pleural or pericardial effusion is expected to be prolonged with the combined use of osimertinib plus bevacizumab and to demonstrate their safety[26]. Consistent with the above studies, our preclinical experiments and retrospective analysis indicated that osimertinib and bevacizumab improve the survival of LM patients with EGFR mutant NSCLC. In contrast, some reports showed that TKIs combined with angiogenesis inhibitors did not improve PFS in EGFR-mutant NSCLC[27]. Although the overall response rate (ORR) was better with osimertinib plus bevacizumab than osimertinib alone (68\% vs $54 \%$ ), median PFS was not longer with osimertinib plus bevacizumab (9.4 months vs 13.5 months)[28]. Dr. Toi reported that compared with osimertinib alone $(n=40)$, the combination of osimertinib and bevacizumab $(n=41)$ did not increase the PFS of NSCLC patients. Although the ORR of the combined group was higher, there was no difference in OS between the two groups[28]. Recent studies have found that $160 \mathrm{mg}$ of osimertinib could have better efficacy than $80 \mathrm{mg}$ in controlling $\operatorname{LM}[8,29,30]$. Further investigation to compare the efficacy of osimertinib 80 to $160 \mathrm{mg}$ in patients with LM is warranted.

VEGF is a key regulator of angiogenesis and a validated target for NSCLC[31]. The biologically synergistic antitumor activity of EGFR inhibition in combination with VEGF/VEGFR pathway blockade have been demonstrated in preclinical studies[32]. In EGFR-mutant NSCLCs, up-regulated EGFR signaling increases VEGF through hypoxia-independent mechanisms, and elevated VEGF, in turn, contributes to the emergence of resistance to EGFR tyrosine kinase inhibitors (TKIs) [33, 34], and EGFR, similar to VEGFR-2, can be expressed on tumor-associated endothelial cells[23,32]. The inhibitory effects of afatinib on EMT and tumorigenesis may be associated with the ERK-VEGF/MMP9 signaling pathway[35]. The TMD has been regarded as one important indicator for quantitatively analyzing tumor angiogenesis, which can clearly reflect the intra-tumoral blood vessels state and tumor-induced angiogenesis ability[36, 37]. Tumor vascularization is critical to the pathogenesis of solid tumors, and TMD is related to tumor invasiveness and metastasis formation which could be used as a potential predictive marker for bevacizumab benefit[38]. Bevacizumab prunes vessels while normalizing those remaining[39]. Osimertinib is a small molecule targeted drug, which is easier to penetrate the blood-brain barrier than the first and second- 
generation targeted drugs[17]. These studies suggest that dual blockade of the VEGF and EGFR pathways would be more effective. Our results showed that the combination group has a more significant reduction in TMD. Perhaps it is the reason that bevacizumab improved the concentrations of osimertinib in the mouse brain, which further indicated that osimertinib plus bevacizumab have a synergistic effect. The further mechanism still needs to be investigated. Considering, our study found that the combined treatment significantly increased the effective intracranial concentration of osimertinib, modulated the level of E-cadherin and downregulated the levels of EGFR and downstream signaling pathways including p-AKT and reduced TMD, indicated that combined osimertinib with bevacizumab could play a synergistic effect in EGFR-mutant LM model possibly by modulating the level of E-cadherin. In preclinically, osimertinib combined with bevacizumab is a more ideal and optimized treatment plan for EGFR mutant NSCLC with LM.

Although our research is instructive for the clinical treatment of NSCLC patients with LM. Owing to the animal model and the small number of patients, this study may not be conclusive. First, strictly speaking, our LM model belongs to a local growth model not metastasis model. Currently, there is no stable disease model that metastasizes to the meninges via the primary tumor. Commonly used models including cisternal injection and lateral ventricle injection, both of which are spread to the meninges through the cerebrospinal fluid circulation route. They have not been metastasized from the primary focus; hence they are not suitable for the study of the mechanism of LM. However, due to their excellent performance stability, it is benefit for drug intervention experiments. Second, it is a small sample size retrospective study, and there are several patients on survival. It is difficult to assess the iPFS of LM, and it is currently believed that a comprehensive assessment should be based on patient neurological examination, $C$ radiological evaluation, and cerebrospinal fluid cytology. The main challenge is to define measurable and non-measurable (target) damage, and allow assessment of response changes, perform cerebrospinal fluid cytology for assessment, due to lumbar puncture is an invasive test, most patients refuse to perform. Therefore, it is difficult and subjective to evaluate iPFS in patients with $L M$, and the present results must be interpreted cautiously. The data were obtained from medical files, and we cannot exclude the possibility of undefined biases and/or confounding factors. Third, the interaction mechanism of osimertinib and bevacizumab needed to be further explored. The next step of our research, the phase II study of osimertinib plus bevacizumab for LM is already ongoing (NCT04425681). We are collecting the CSF and blood of NSCLC patients with LM in osimertinib group and the combination with bevacizumab group to further explore the mechanism and find the biomarkers for prognostic.

In conclusion, the current findings demonstrated that osimertinib plus bevacizumab tend to improve the outcome than osimertinib for LM patients with EGFR-mutant NSCLC, suggesting osimertinib plus bevacizumab is an appropriate treatment option for patients with LM from EGFR mutant NSCLC.

\section{Abbreviations}

\section{EGFR}

Epidermal growth factor receptor 
H\&E

Hematoxylin-eosin staining

iPFS

intracranial progressive-free survival

IVIS

Interactive Video Information System

LM

Leptomeningeal metastasis

MRI

Magnetic Resonance Imaging

NSCLC

Non-small cell lung cancer

TKIs

Tyrosine kinase inhibitors

TMD

Tumor microvessel density

OS

Overall survival

\section{Declarations}

\section{Ethics approval and consent to participate}

The authors are accountable for all aspects of the work in ensuring that questions related to the accuracy or integrity of any part of the work are appropriately investigated and resolved. All animal experiments were approved by the Ethical Committee on Animal Experiments in the Second Affiliated Hospital of Nanchang University (NO.: Review [2019] No. (033)), in compliance with Chinese national or institutional guidelines for the care and use of animals. The study for retrospective experiments was approved by institutional board of The Second Affiliated Hospital of Nanchang University (NO.: Review [2019] No. (032)) and individual consent for this retrospective analysis was waived. The study was conducted in accordance with guidelines from Animal Research: Reporting of In Vivo Experiments (ARRIVE) and all methods were carried out in accordance with relevant guidelines and regulations.

\section{Consent for publication}

Not Applicable

\section{Availability of data and material}

All data generated or analyzed during this study are included in this published article.

\section{Competing interests}


The authors declare they have no conflicts of interest relevant to this submission.

\section{Funding}

This research was supported by the National Natural Science Foundation of China [grant number 81760566, 82060577], Key Research and Development Project of Jiangxi province [grant number 20181ACG70011].

\section{Authors' contributions}

YL: Data curation, Formal analysis, Investigation, Methodology, Software, Validation, Visualization, Writing-original draft, Writing-review \& editing. JC: Formal analysis, Validation, Visualization, Writing review \& editing. PX: Formal analysis, Methodology, Validation, Visualization, Writing review \& editing. LX: Validation, Visualization, Writing review \& editing. ZQ: Formal analysis, Methodology, Software, Validation, Writing review \& editing. ZM: Data curation, Project administration, Resources, Validation, Writing-original draft, Writing-review \& editing. AW: Conceptualization, Data curation, Formal analysis, Funding acquisition, Investigation, Methodology, Resources, Software, Supervision, Validation, Visualization, Writing review \& editing. All authors have read and approved the manuscript.

\section{Acknowledgements}

We are very grateful to Dr. He Yuanqiao's team for their technical guidance on animal experiments, and to Director Huang Xiaoning for his generous help in imaging.

\section{References}

1. Siegel, R.L., K.D. Miller, and A. Jemal, Cancer statistics, 2019. CA: a cancer journal for clinicians, 2019. 69(1).

2. Li, Y., et al., Leptomeningeal Metastases in Patients with NSCLC with EGFR Mutations. Journal of thoracic oncology : official publication of the International Association for the Study of Lung Cancer, 2016. 11(11): p. 1962-1969.

3. Taillibert, S. and M.C. Chamberlain, Leptomeningeal metastasis. Handbook of clinical neurology, 2018. 149: p. 169-204.

4. Gourd, E., Osimertinib for leptomeningeal metastases in NSCLC. The Lancet. Oncology, 2020. 21(1): p. e17.

5. H, C. and P.-S. R, Leptomeningeal metastases in non-small-cell lung cancer. The Lancet. Oncology, 2018. 19(1): p. e43-e55.

6. Boire, A., et al., Complement Component 3 Adapts the Cerebrospinal Fluid for Leptomeningeal Metastasis. Cell, 2017. 168(6): p. 1101-1113 e13.

7. Remon, J., E. Le Rhun, and B. Besse, Leptomeningeal carcinomatosis in non-small cell lung cancer patients: A continuing challenge in the personalized treatment era. Cancer treatment reviews, 2017. 
53: p. 128-137.

8. Park, S., et al., A phase II, multicenter, two cohort study of 160 mg osimertinib in EGFR T790Mpositive non-small-cell lung cancer patients with brain metastases or leptomeningeal disease who progressed on prior EGFR TKI therapy. Annals of oncology : official journal of the European Society for Medical Oncology, 2020. 31(10): p. 1397-1404.

9. Ahn, M.-J., et al., Osimertinib for Patients With Leptomeningeal Metastases Associated With EGFR T790M-Positive Advanced NSCLC: The AURA Leptomeningeal Metastases Analysis. Journal of thoracic oncology : official publication of the International Association for the Study of Lung Cancer, 2019.

10. Di Maio, M., et al., Combining anti-Epidermal Growth Factor Receptor (EGFR) and anti-angiogenic strategies in advanced NSCLC: we should have known better... Current pharmaceutical design, 2014. 20(24): p. 3901-3913.

11. H, S., et al., Erlotinib plus bevacizumab versus erlotinib alone in patients with EGFR-positive advanced non-squamous non-small-cell lung cancer (NEJ026): interim analysis of an open-label, randomised, multicentre, phase 3 trial. The Lancet. Oncology, 2019. 20(5): p. 625-635.

12. Yu, H.A., et al., Effect of Osimertinib and Bevacizumab on Progression-Free Survival for Patients With Metastatic EGFR-Mutant Lung Cancers: A Phase 1/2 Single-Group Open-Label Trial. JAMA oncology, 2020. 6(7): p. 1048-1054.

13. Lu, Z.-Q., et al., Osimertinib combined with bevacizumab for leptomeningeal metastasis from EGFRmutation non-small cell lung cancer: A phase Il single-arm prospective clinical trial. Thoracic cancer, 2020.

14. Le Rhun, E., et al., EANO-ESMO Clinical Practice Guidelines for diagnosis, treatment and follow-up of patients with leptomeningeal metastasis from solid tumours. Annals of oncology : official journal of the European Society for Medical Oncology, 2017. 28: p. iv84-iv99.

15. D, K., et al., A model of breast cancer meningeal metastases: characterization with in vivo molecular imaging. Cancer gene therapy, 2019. 26(null): p. 145-156.

16. Xiong, B., et al., Precise Cerebral Vascular Atlas in Stereotaxic Coordinates of Whole Mouse Brain. Frontiers in neuroanatomy, 2017. 11: p. 128.

17. Cross, D.A., et al., AZD9291, an irreversible EGFR TKI, overcomes T790M-mediated resistance to EGFR inhibitors in lung cancer. Cancer Discov, 2014. 4(9): p. 1046-61.

18. Ilhan-Mutlu, A., et al., Bevacizumab Prevents Brain Metastases Formation in Lung Adenocarcinoma. Molecular cancer therapeutics, 2016. 15(4): p. 702-710.

19. Zeng, Z.-M., et al., BRCA1 protects cardiac microvascular endothelial cells against irradiation by regulating p21-mediated cell cycle arrest. Life sciences, 2020. 244: p. 117342.

20. Paschalis, A., et al., Prostate-specific Membrane Antigen Heterogeneity and DNA Repair Defects in Prostate Cancer. European urology, 2019. 76(4): p. 469-478.

21. Detre, S., G. Saclani Jotti, and M. Dowsett, $A$ "quickscore" method for immunohistochemical semiquantitation: validation for oestrogen receptor in breast carcinomas. Journal of clinical 
pathology, 1995. 48(9): p. 876-8.

22. Zhao, B., et al., Efficacy and safety of therapies for EGFR-mutant non-small cell lung cancer with brain metastasis: an evidence-based Bayesian network pooled study of multivariable survival analyses. Aging, 2020. 12(14): p. 14244-14270.

23. Le, X., et al., Dual EGFR-VEGF Pathway Inhibition: A Promising Strategy for Patients With EGFRMutant NSCLC. Journal of thoracic oncology : official publication of the International Association for the Study of Lung Cancer, 2020.

24. Seto, T., et al., Erlotinib alone or with bevacizumab as first-line therapy in patients with advanced nonsquamous non-small-cell lung cancer harbouring EGFR mutations (JO25567): an open-label, randomised, multicentre, phase 2 study. The Lancet. Oncology, 2014. 15(11): p. 1236-1244.

25. Kato, T., et al., Erlotinib Plus Bevacizumab Phase II Study in Patients with Advanced Non-small-Cell Lung Cancer (JO25567): Updated Safety Results. Drug safety, 2018. 41(2): p. 229-237.

26. Hiranuma, O., et al., Rationale and Design of a Phase II Trial of Osimertinib Combined With Bevacizumab in Patients With Untreated Epidermal Growth Factor Receptor-mutated Non-small-cell Lung Cancer and Malignant Pleural and/or Pericardial Effusion (SPIRAL I/ Study). Clinical lung cancer, 2019. 20(3): p. e402-e406.

27. Stinchcombe, T.E., et al., Effect of Erlotinib Plus Bevacizumab vs Erlotinib Alone on Progression-Free Survival in Patients With Advanced EGFR-Mutant Non-Small Cell Lung Cancer: A Phase 2 Randomized Clinical Trial. JAMA oncology, 2019.

28. Akamatsu, H., et al., Efficacy of Osimertinib Plus Bevacizumab vs Osimertinib in Patients With EGFR T790M-Mutated Non-Small Cell Lung Cancer Previously Treated With Epidermal Growth Factor Receptor-Tyrosine Kinase Inhibitor: West Japan Oncology Group 8715L Phase 2 Randomized Clinical Trial. JAMA oncology, 2021. 7(3): p. 386-394.

29. Saboundji, K., et al., Efficacy of Osimertinib in EGFR-Mutated Non-Small Cell Lung Cancer with Leptomeningeal Metastases Pretreated with EGFR-Tyrosine Kinase Inhibitors. Targeted oncology, 2018. 13(4): p. 501-507.

30. Lee, J., et al., Osimertinib Improves Overall Survival in Patients With EGFR-Mutated NSCLC With Leptomeningeal Metastases Regardless of T790M Mutational Status. Journal of thoracic oncology : official publication of the International Association for the Study of Lung Cancer, 2020. 15(11): $p$. 1758-1766.

31. Nilsson, M. and J.V. Heymach, Vascular endothelial growth factor (VEGF) pathway. Journal of thoracic oncology : official publication of the International Association for the Study of Lung Cancer, 2006. 1(8): p. 768-770.

32. Byers, L. and J. Heymach, Dual targeting of the vascular endothelial growth factor and epidermal growth factor receptor pathways: rationale and clinical applications for non-small-cell lung cancer. Clinical lung cancer, 2007: p. S79-85.

33. Swinson, D. and K. O'Byrne, Interactions between hypoxia and epidermal growth factor receptor in non-small-cell lung cancer. Clinical lung cancer, 2006. 7(4): p. 250-6. 
34. Le, X., et al., Dual EGFR-VEGF Pathway Inhibition: A Promising Strategy for Patients With EGFRMutant NSCLC. Journal of thoracic oncology : official publication of the International Association for the Study of Lung Cancer, 2021. 16(2): p. 205-215.

35. Chen, Y., et al., Afatinib, an EGFR inhibitor, decreases EMT and tumorigenesis of Huh-7 cells by regulating the ERK-VEGF/MMP9 signaling pathway. Molecular medicine reports, 2019. 20(4): $\mathrm{p}$. 3317-3325.

36. Shang, F., et al., The anti-angiogenic effect of dexamethasone in a murine hepatocellular carcinoma model by augmentation of gluconeogenesis pathway in malignant cells. Cancer chemotherapy and pharmacology, 2016. 77(5): p. 1087-96.

37. Hasan, J., R. Byers, and G. Jayson, Intra-tumoural microvessel density in human solid tumours. British journal of cancer, 2002. 86(10): p. 1566-77.

38. Bais, C., et al., Tumor Microvessel Density as a Potential Predictive Marker for Bevacizumab Benefit: GOG-0218 Biomarker Analyses. Journal of the National Cancer Institute, 2017. 109(11).

39. Tolaney, S., et al., Role of vascular density and normalization in response to neoadjuvant bevacizumab and chemotherapy in breast cancer patients. Proceedings of the National Academy of Sciences of the United States of America, 2015. 112(46): p. 14325-30.

\section{Figures}


A

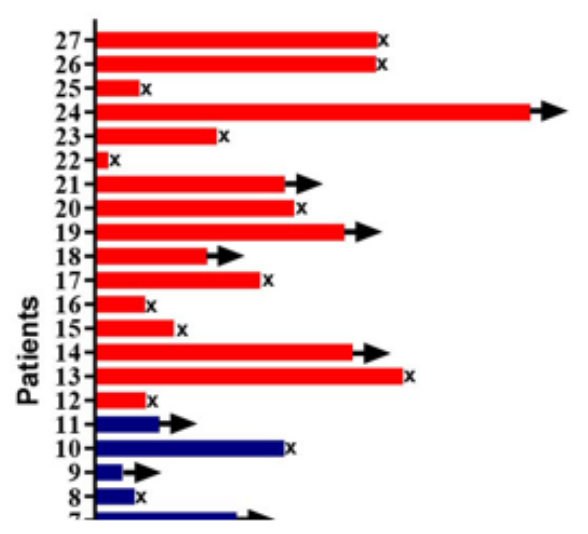

B

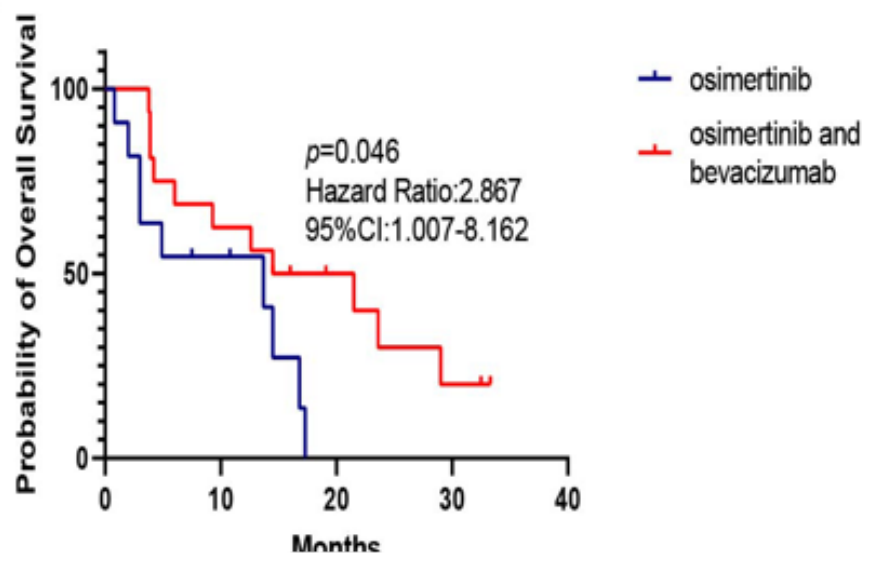

Figure 1

Swimmier plot and OS and iPFS curve of LM patients with EGFR mutant NSCLC

Figure 2

Construction of LM model and short-term oral absorption test in vivo

Figure 3

Antitumor activity of osimertinib and bevacizumab in the EGFR-mutant LM model

Figure 4 
Osimertinib plus bevacizumab suppresses the EGFR downstream signaling pathway and reduce the TMD

Figure 5

Osimertinib plus bevacizumab modulates E-cadherin levels in EGFR-mutant LM model mice

\section{Supplementary Files}

This is a list of supplementary files associated with this preprint. Click to download.

- Supplementarytable.docx 